\title{
Quadruped Robot Navigation Considering the Observational Cost
}

\author{
Takeshi Fukase, Masahiro Yokoi, Yuichi Kobayashi, Ryuichi Ueda, \\ Hideo Yuasa, and Tamio Arai \\ The Department of Precision Engineering, The University of Tokyo, Hongo, \\ Bunkyo-ku, Tokyo, 113-8656, Japan \\ \{fukase, yokoi, kobayashi, ueda, yuasa, arai\}@prince.pe.u-tokyo.ac.jp
}

\begin{abstract}
In this paper, we present a new method to decide the optimal robot's motion with a quadruped robot of Sony Legged Robots League. The method keeps the "observational cost" as small as possible. The task we cope with is navigation. The robot can reach the destination rapidly and robustly with this method in spite of under the unfavorable conditions such as restricted sensor ability, limited CPU power, and inaccurate locomotion. Finally the efficiency of our method is verified by experiments.
\end{abstract}

\section{Introduction}

On the soccer field, the robot's decision mainly depends on its location. There are many researches about localization[1, 2, 3]. However, the task which should be finally accomplished is not to localize accurately, but to decide the optimal motion.

There are two researches which provide effective motion decisions with simple observations under the condition of Sony Legged Robots League 4, 5]. In these researches, the robot decides its motion according to the appearance of the landmarks and the ball. However, the relations between the location and the decision are designed arbitrarily. Therefore, the optimality of the decision is not guaranteed.

In this paper, we propose a method to decide the optimal motion with the smallest observational cost. The task we cope with is navigation. This is a task to make a robot reach a target position and orientation which has some allowance called "target region". This task is applicatory to other tasks such as occupying a defensive position. In addition, we can evaluate the optimality by the time to reach the target region.

In this research, we use ERS-1100 that is the unique hardware in Sony Legged Robot League. Following characteristics of ERS-1100 make the task difficult.

- Restricted sensor ability: Insufficient information due to the narrow field of view

- Limited CPU power: A calculation that needs high CPU power is not executable. 
- Limited control ability: The discrete locomotion is not adapted for a small move.

The approaches to accomplish the navigation are as follows. First, we use "Motion Planned Map(MPM)" to decide the optimal locomotion commands. Second, as a localization method, we adopt "Sensor Resetting Localization[3] (SRL)" which is developed for Sony Legged Robots League. By using "MPM" and "SRL", we propose a method that provides the motion decision that minimizes the observational cost.

The rest of this paper is constructed as follows; Section 2 discusses the control method we propose in this research. Section 3 provides the results of the experiments and discussions. Section 4 provides the conclusion and future works.

\section{Methods}

Here we summarize our method to navigate a robot to the target region(Fig (1). At the beginning of every step, the robot's estimated position is updated by SRL. Then, the robot decides its motion by comparing the cost of observation with the risk of moving without observation according to its estimated position and MPM. And it executes the motion. This process is repeated until the robot reaches the target region.

\subsection{Motion Planned Map}

MPM is a data base which has the information of optimal locomotion commands at any robot's position and orientation. We make this Map by the offline calculation. Therefore, only a low computational power is required to the robot. In this research, the map is represented by grid cells. We made this map with Dynamic Programming. Fig 2 shows the MPM made under the conditions shown in Table 1 .

When making MPM, we can also calculate the expectations of the time from each grid to the target region. We express the position-time relation by gridbased form which is the same as MPM. We call this "State Value Map (SVM)", and use with the MPM.

\subsection{Motion Decision Method}

After updating the self-position, the motion to be executed is decided according to MPM. In SRL, a set of finite discrete points called samples represents the positional and orientational probabilistic distribution of the robot. When all SRL samples are inside the single region as shown in Fig.3(a), the motion which corresponds to the region on the map is selected. On the other hand, we must decide to move or observe when the samples are distributed over more than two regions as shown in Fig[3(b). This decision is made according to the rule shown in Fig 4. That is "If the observational cost is smaller than the loss of time caused 
by non-optimal decision, then we decide to observe". The expectation of the time to the target region is calculable by the samples' positions and orientation and the value of the SVM there.

\section{$3 \quad$ Experiments}

\subsection{Conditions of Experiments}

The described method was implemented and evaluated under four different conditions shown in Table 2, When the initial position is "fixed and known", all the samples are settled on the actual robot's position on the soccer field. On the other hand, "random and unknown" means that the samples are distributed uniformly.

In addition, we set other conditions as follows:

- Initial position and Target region: Shown in Fig 5.

- Number of samples: 100(fixed).

- Observational cost: $3.0[\mathrm{sec}]$ (fixed).

- Number of trials: 10 (on each condition)

- Terminative condition: Beyond $80 \%$ of all the samples are inside the target region.

- Judgment of success: The robot actually reaches the target region in $180[\mathrm{sec}]$.

\subsection{Result and Discussions}

Table.3 shows the result of the experiments. Fig 6 shows the overview of the experiments.

The robot successfully reached the target region at the rate of about $70 \%$ on each condition. In Experiment.1 and 3, the numbers of observations were only three or four times. In Experiment 2 and 4, they were larger. This means that the robot selected to observe according to its positional and orientational probabilistic distribution.

In Experiment.1 and 3, the initial position and orientation were the same on each trial. However, there were no two trials on which the robot crossed the same path to the target region. This is due to the errors of robot's locomotion and the localization. The fact that the robot can reach the target region through different paths indicates the robustness of our method.

In failure cases, the robot considered itself inside the target region based on the distribution of samples even though it is located incorrect actual position. This is due to the factors which are unconsidered or unable to model in SRL such as the collision with the walls. In order to improve the success rate, we must clear up the causes of localization errors and model them. This is also an essential problem. One supposable approach is the practical use of information about other objects in addition to the landmarks. 


\section{Conclusion and Future Works}

In this research, we proposed the method to decide the optimal motion with the smallest observational cost. We need not to set the arbitrary rules to decide the robot's motions. The robot can select the optimal motion in spite of the uncertain information of its location. The efficiency of our method was verified by the results of the experiments.

In this method, it is assumed that the probabilistic distribution of the location is modified well enough to select the optimal locomotion by an observation. But the observation causes the loss of time if the robot is located where the recognition of the landmarks is impossible. In this case, it is necessary to predict the efficiency of the observation. In future, the prediction of the observational efficiency should be considered. Furthermore, improvement of localization method and tasks with the ball or dynamic obstacles also will be our future works.

\section{References}

[1] P. Buschka, A. Saffiotti and Z. Wasil: "Fuzzy Landmark-Based Localization for a Legged Robot", Proc. International Conference on Intelligent Robots and Systems, pp.1205-1210, (2000)

[2] Masahiro YOKOI, Yuichi KOBAYASHI, Takeshi FUKASE, Hideo YUASA and Tamio ARAI: "Learning Self-Localization with Teaching System", Proc. International Conference on Intelligent Robots and Systems, pp.1211-1216, (2000)

[3] S. Lenser and M. Veloso: "Sensor Resetting Localization for Poorly Modeled Mobile Robot", Proc. International Conference on Robotics and Automation, pp.12251232, (2000)

[4] Vincent HUGEL, Patrick BONIN and Pierre BLAZEVIC: "Reactive and Adaptive Control Architecture Designed for the Sony Legged Robots League in RoboCup 1999", Proc. International Conference on Intelligent Robots and Systems, pp.1211$1216,(2000)$

[5] Noriaki Mitsunaga and Minoru Asada: "Observation strategy for decision making based on information criterion", Proc. International Conference on Intelligent Robots and Systems, pp.1211-1216, (2000)

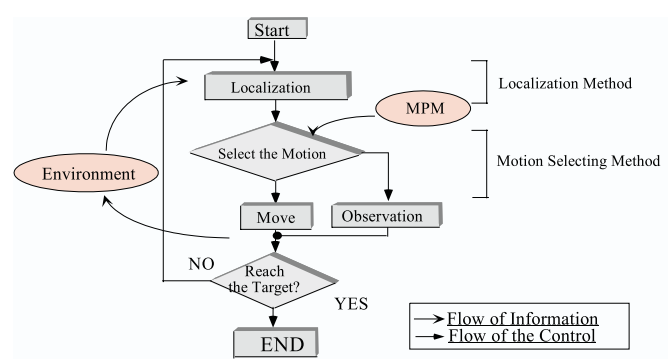

Fig. 1. The flow chart of the control method. 
Table 1. The conditions to make Motion Planned Map

\begin{tabular}{cc}
\hline Locomotion commands & Forward, Right turn, Left turn $(3$ motions $)$ \\
Map size & $5000[\mathrm{~mm}] \times 5000[\mathrm{~mm}] \times 360[\mathrm{deg}]$ \\
Grid size & $50[\mathrm{~mm}] \times 50[\mathrm{~mm}] \times 15[\mathrm{deg}]$ \\
Target region & $(2500 \pm 300[\mathrm{~mm}], 2500 \pm 300[\mathrm{~mm}], 0 \pm 30[\mathrm{deg}]$ \\
\hline
\end{tabular}

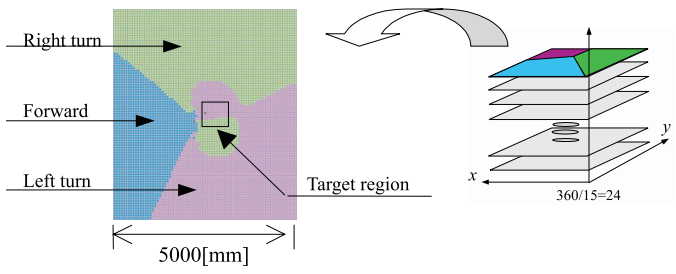

Fig. 2. Motion Planned Map. This map shows the result between $0[\mathrm{deg}]$ and $15[\mathrm{deg}]$. Actually each orientation creates different maps. That means we get 24 maps.

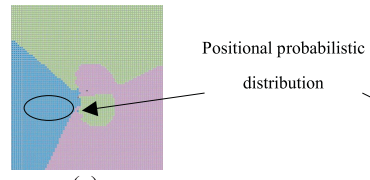

(a)

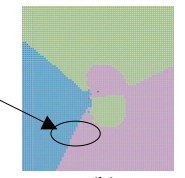

(b)

Fig. 3. The motion decision method with the positional probabilistic distribution. In case of (a), the robot move "Forward", but in case of (b), it is needed to decide to move or observe.

\section{Variables}

$\begin{array}{llll}\text { Number of the locomotion command } & : n & \\ \text { ID of the locomotion commands } & :{ }^{i} & \\ \text { Locomotion command } & : a_{i} & E_{0}=\sum_{j=0}^{N} p_{j, 0} v_{j, 0} \\ \text { Cost of the locomotion } & : t_{i} & E_{i}=\sum_{j=0}^{N} p_{j, i} v_{j, i} \\ \text { Cost of the observation } & : t_{\text {observation }} & t_{\max }=\max _{1 \leq i \leq n}\left(E_{i}-E_{0}+t_{i}\right) \\ \text { Set of samples of the initial state } & : \Omega_{0} & t_{\min }=\min _{1 \leq i \leq n}\left(E_{i}-E_{0}+t_{i}\right) \\ \text { Set of samples after the locomotion } a_{i} & : \Omega_{i} & d=\arg \min _{1 \leq i \leq n}\left(E_{i}-E_{0}+t_{i}\right) \\ \text { Number of samples } & :{ }^{N} & \text { if }\left(t_{\max } \leq t_{\text {observation }}\right) \text { do } a_{d} \text { locomotion } \\ \text { Sample } j \text { at the initial state } & : \omega_{j, 0} & \text { if }\left(t_{\max } \geq t_{\text {observation }}\right) \text { do observation } \\ \text { Sample } j \text { after the locomotion } a_{i} & : \omega_{j, i} & \\ \text { SVM value of } \omega_{j, 0} & : v_{j, 0} & \\ \text { SVM value of } \omega_{j, i} & v_{j, i} & \\ \text { Expectation from } \Omega_{0} \text { to the target region } & : E_{0} & \\ \text { Expectation from } \Omega_{i} \text { to the target region }: & E_{i}\end{array}$

Fig. 4. The motion selecting algorism. 
Table 2. Conditions of experiments

\begin{tabular}{lcc}
\hline & Locomotion patterns & Initial position \\
Experiment.1 & 3 & fixed and known \\
Experiment.2 & 3 & random and unknown \\
Experiment.3 & 5 & fixed and known \\
Experiment.4 & 5 & random and unknown \\
\hline
\end{tabular}

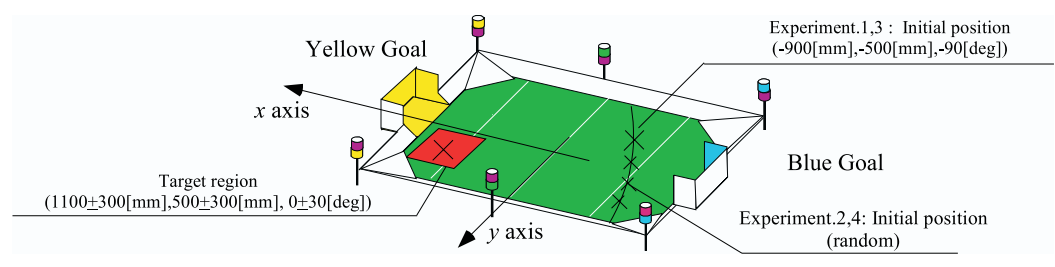

Fig. 5. The initial position and target region of this experiment.

Table 3. The results of the experiments.

\begin{tabular}{lccc}
\hline & Succeed times & Average of the time[sec] & The number of observation \\
Experiment.1 & $7 / 10$ & 62.3 & 3.4 \\
Experiment.2 & $6 / 10$ & 65.2 & 5.0 \\
Experiment.3 & $7 / 10$ & 53.4 & 3.5 \\
Experiment.4 & $7 / 10$ & 100.4 & 7.4 \\
\hline
\end{tabular}

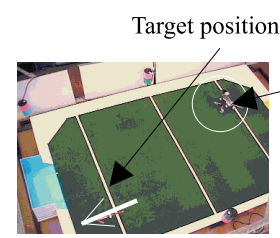

(a)

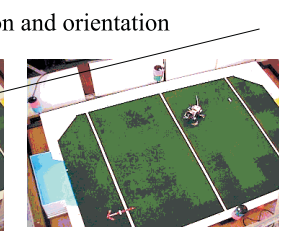

(b)

Initial position

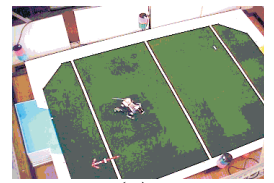

(c)

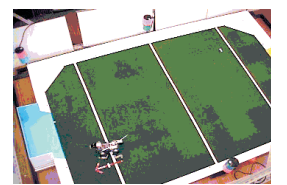

(d)

Fig. 6. The overview of this experiment. 